\title{
Dietary Factors and Prevention: Risk of End-Stage Kidney Disease by Fruit and Vegetable Consumption
}

\author{
Tanushree Banerjee $^{\mathrm{a}} \quad$ Juan Jesus Carrero ${ }^{\mathrm{b}}$ Charles McCulloch ${ }^{\mathrm{c}}$ \\ Nilka Rios Burrows $^{d}$ Karen R. Siegel ${ }^{d}$ Hal Morgenstern ${ }^{\mathrm{e}, f}$ Rajiv Saran ${ }^{g, h}$ \\ Neil R. Powe ${ }^{i}$ on behalf of the Centers for Disease Control and Prevention \\ Chronic Kidney Disease Surveillance Team
}

\begin{abstract}
${ }^{a}$ Division of General Internal Medicine, Department of Medicine, University of California, San Francisco, CA, USA; ${ }^{b}$ Department of Medical Epidemiology and Biostatistics, Karolinska Institutet, Solna, Sweden; 'Department of Biostatistics, University of California, San Francisco, CA, USA; dDivision of Diabetes Translation, Centers for Disease and Control and Prevention, Atlanta, GA, USA; ${ }^{e}$ Departments of Epidemiology and Environmental Health Sciences, School of Public Health, University of Michigan, Ann Arbor, MI, USA; 'Department of Urology, Medical School, University of Michigan, Ann Arbor, MI, USA; ${ }^{9}$ Kidney Epidemiology \& Cost Center, University of Michigan, Ann Arbor, MI, USA; hDivision of Nephrology, Department of Medicine, University of Michigan, Ann Arbor, MI, USA; 'Department of Medicine, Zuckerberg San Francisco General Hospital, San Francisco, CA, USA
\end{abstract}

\begin{abstract}
Background: The association between fruit and vegetable (FV) intake and the risk of end-stage kidney disease (ESKD) has not been examined in the general population and fully explored in chronic kidney disease (CKD). We prospectively evaluated this relationship in US representative sample of adults and evaluated consistency by the presence or absence, and severity, of CKD. Methods: We used data from the Third National Health and Nutrition Examination Survey (1988-1994) linked with the US Renal Data System, including 14,725 adults aged $\geq 20$ years and with follow-up for ESKD through 2008. Daily FV intake was ascertained using a food frequency questionnaire. We examined the association between selected categories of FV intake and ESKD using a Fine Gray competing risk model adjusting for sociodemographics, lifestyle, clinical and nutritional factors, estimated glomerular filtration rate, and albuminuria. We evaluated whether risk varied in individuals with severe versus any
\end{abstract}

CKD. Results: 230 participants (1.5\%) developed ESKD during follow-up. In the adjusted model, compared to highest intake, those in lowest categories of FV intake had a higher risk of ESKD, for $<2$ times/day (1.45 [1.24-1.68], 2 to $<3$ times/ day (1.40 [1.18-1.61]), 3 to $<4$ times/day (1.25 [1.04-1.46]), and 4 to $<6$ times/day (1.14 [0.97-1.31]). There was suggestion of heterogeneity ( $p$ for interaction $=0.03$ ) with possible stronger inverse association in patients with CKD than those without CKD. After stratification, we obtained similar strong inverse association when we examined ESKD incidence across intake of FVs in participants with CKD stages 1-4 ( $n=$ $5,346)$ and specifically in those with CKD stages 3-4 $(n=$ 1,084). Conclusions: Low intake of FVs was associated with higher risk of ESKD in US adults with and without CKD, supporting an emerging body of literature on the potential benefits of plant-rich diets for prevention of ESKD.

(C) 2021 The Author(s)

Published by S. Karger AG, Basel
(C) 2021 The Author(s)

Published by S. Karger AG, Basel

This is an Open Access article licensed under the Creative Commons Attribution-NonCommercial-4.0 International License (CC BY-NC) (http://www.karger.com/Services/OpenAccessLicense), applicable to the online version of the article only. Usage and distribution for commercial purposes requires written permission.
Correspondence to:

Tanushree Banerjee, tanushree.banerjee@ucsf.edu 


\section{Introduction}

Chronic kidney disease (CKD) is a prevalent complex disease and is fast becoming a growing public health problem, leading to premature mortality or poor quality of life, imposing a heavy burden on health-care systems $[1,2]$. Available evidence reveals that the global prevalence of CKD has a sharply increasing trend from 2000 to 2016, affecting $10-15 \%$ of general populations [3]. Aging, hypertension, diabetes, hyperlipidemia, smoking, and poor dietary intake have been identified as risk factors for CKD occurrence $[4,5]$. There is relatively little knowledge as to how to prevent CKD and most of the recommendations stem from lifestyle modifications extrapolated from lowering blood pressure and cardiovascular disease (CVD) risk [6,7], which is lowering the intake of salt.

Diet may influence the occurrence of CKD. Studies examining adherence to a Mediterranean diet or a Dietary Approaches to Stop Hypertension (DASH) diet, or patterns of diet consistent with vegetarian, pro-vegetarian or healthy plant-based foods in the general population have shown consistent associations with a lower CKD prevalence and a lower incidence rate of CKD $[8,9]$. Emerging evidence in patients with CKD suggests these diets may be helpful to delay progression to end-stage kidney disease (ESKD) and prevent complications [10]. Both the diets encourage whole foods, such as fruits, vegetables, beans/legumes, whole grains, and nuts with a restriction on the amount of animal protein consumed. However, it is not known whether a single component of these diets, that is, a higher intake of fruits and vegetables (FVs), associates with the lower risk of ESKD. The effectiveness of an increasing FV consumption has only been tested in selected patients with CKD stages 3-4 with hypertension and macroalbuminuria in a small trial, and FV intake was found to be as effective as sodium bicarbonate in delaying CKD decline $[12,13]$. Despite such a positive association noted in individuals with advanced CKD, consuming FVs is traditionally discouraged in this group as a hyperkalemia prevention strategy [11]. The existence of an association between higher FV intake and lower risk of ESKD may inform patient discussions in clinical practice around lifestyle modifications for primary prevention of CKD and secondary disease prevention of CKD such as CVD protection and type 2 diabetes. Delayed progression of CKD has major beneficial effects on both the patient and health-care system. Studies on the general population examining the impact of FV intake on the risk of ESKD have not been conducted, a mini review of the cohort studies available in literature is listed under Table 1.
This study aimed to examine the association between daily number FV intake at baseline and the incidence rate of ESKD in a US representative sample of adults. As a primary hypothesis, we evaluated whether associations differed among individuals with and without baseline CKD and whether they differ for those with severe CKD.

\section{Materials and Methods}

\section{Study Design and Population}

We analyzed a cohort using data from the Third National Health and Nutrition Examination Survey (NHANES III) that were linked with the United States Renal Data System (USRDS) and Mortality files by the Centers for Disease Control and Prevention's National Center for Health Statistics (NCHS) $(n=18,825$ adults). NHANES III is a nationally representative probability survey of US noninstitutionalized civilians conducted between 1988 and 1994 by the NCHS. We excluded 1,225 NHANES III adults younger than 20 years of age, 61 participants with missing dietary information, 105 participants who were pregnant, and 2,709 participants were ineligible for linkage resulting in an analytic sample of 14,725. The linked NHANES III-USRDS-Mortality files contain information on participants diagnosed with ESKD and mortality follow-up data from the start of NHANES III participation to the end of the linkage interval. The ESKD data used were from the Combined ESKD Patient Profile and Death Notification (form 2746) File. This information was based on the results from linking NHANES III participant data, USRDS administrative records, and the National Death Index (NDI) death certificate records (based on probabilistic techniques), the details of which are provided elsewhere $[14,15]$. We chose the criteria for linkage defined for the USRDS records as they were more stringent than the criteria defined for NDI records. The linkage eligibility variable (CMS_Medicare_Match) in the NCHS-CMS Medicare feasibility file was used and participants were linked to the USRDS records if the linkage eligibility variable took the value 1 (i.e., for linkage eligibility and linked).

\section{Sociodemographic and Clinical Measurements}

Medical history and demographic data were collected through a standardized questionnaire conducted at the participant's home followed by a medical examination and laboratory testing that occurred in a mobile examination center [16]. Sociodemographic factors were also assessed during the interview. Racial/ethnic categories were self-reported by NHANES III participants and assigned as non-Hispanic white (NHW), non-Hispanic black (NHB), Mexican American (MA), or other race/ethnicities. Self-reported information on socioeconomic status (SES), that is, education and income, were also included. Self-reported income was assessed using the ratio of household income to the US household poverty level (poverty income ratio) [16].

Diabetes (other than during pregnancy) was defined by measured hemoglobin A1C (HbA1C) $\geq 6.5 \%$ [17]. Hypertension was defined as a measured average of systolic blood pressure (SBP) $\geq 140 \mathrm{~mm} \mathrm{Hg}[18,19]$. Body mass index (BMI) was calculated as weight in kilograms divided by the measured height in meters squared. A physical activity questionnaire was administered at a 


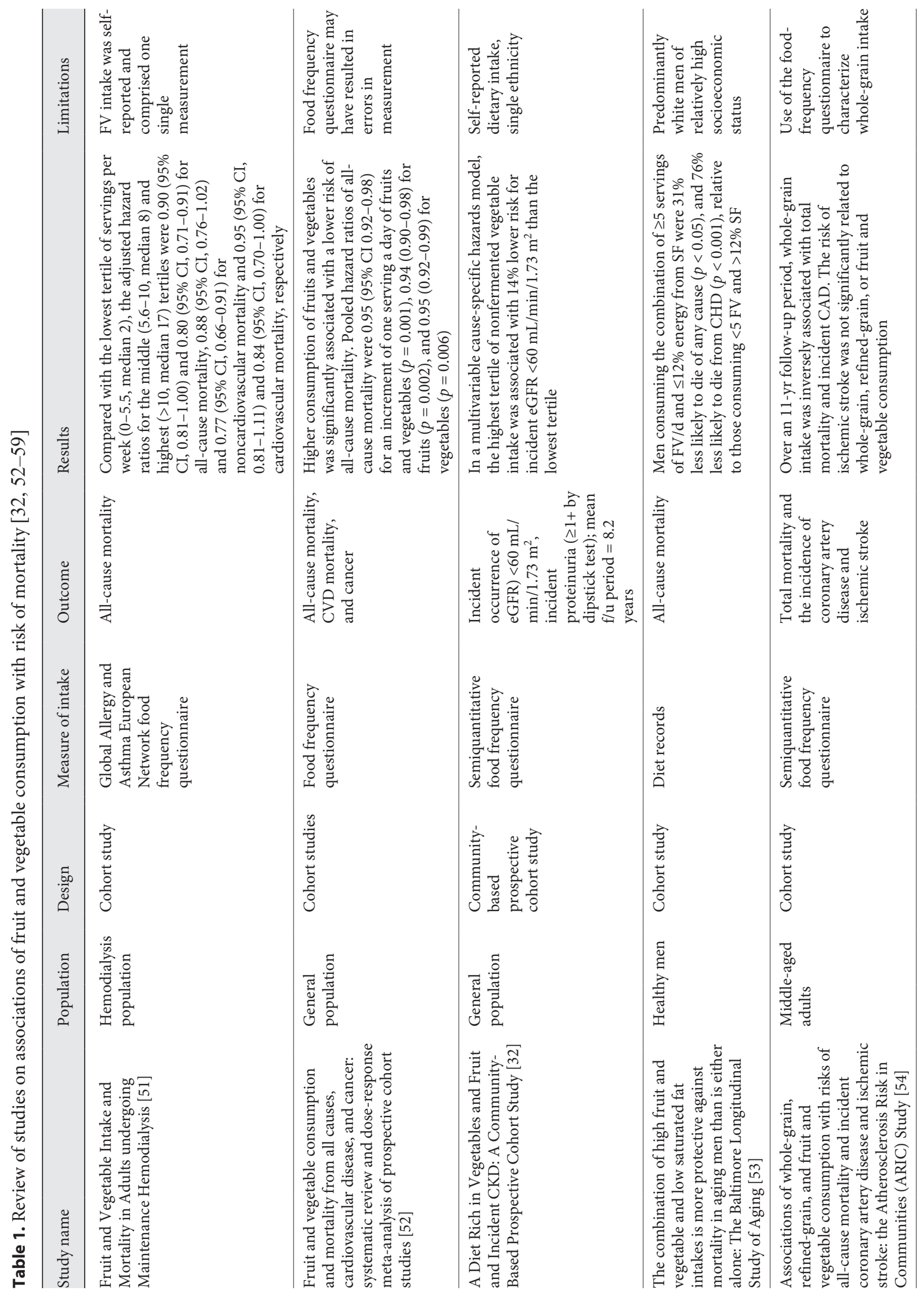



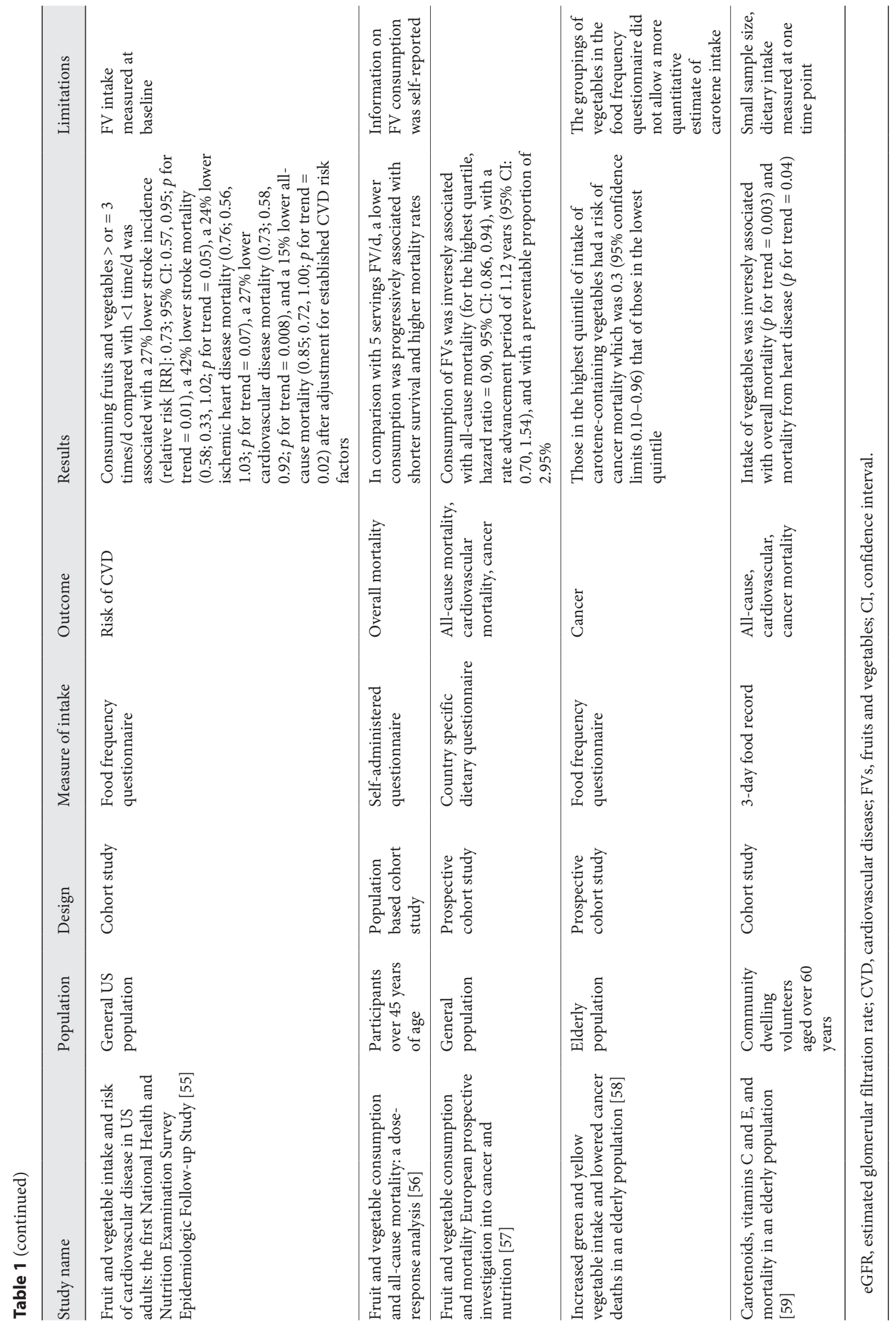
home interview for all participants. They were asked about the frequency of leisure time activity in the past month. This included the frequency of walking a mile without stopping, running or jogging, riding a bicycle or exercise bike, swimming, aerobics, dancing, calisthenics, garden or yard work, lifting weights, or other activities.

\section{FV Intake}

Dietary intake was collected through an in-person food frequency questionnaire (FFQ) conducted by interviewers with a knowledge of food preparation and nutrient composition. Specifically, from the NHANES III FFQ, 2 sections on fruits (questions, namely, HAN3AS, HAN3BS, HAN3CS, HAN3DS, HAN3ES, and HANFS) and vegetables (questions, namely, HAN4AS, HAN4BS, HAN4CS, HAN4DS, HAN4ES, HAN4FS, HAN4GS, HAN4HS, HAN4IS, HAN4JS, HAN4KS, and HAN4LS) asked questions to assess how many times per day, week, or month (over the past 30 days) the participants consumed fruits and vegetables.

Meat and fish intake was determined by the questions asked in the FFQ, such as consumption of (1) bacon/sausage/processed meats; (2) liver and other organ meats; (3) beef; (4) pork and ham; (5) shrimp, clams, etc.; and (6) fish, and was treated as a continuous variable. Daily frequency of intake was calculated by dividing reported intake by 30 for intake reported by month or by 7 for those reporting weekly [20]. Food codes, food weight, and nutrient values were based on data from the US Department of Agriculture, industry, and other sources [21].

\section{Measurement of Albuminuria and Kidney Function and}

Classification of CKD

Serum creatinine measurements obtained using a kinetic rate Jaffé method in NHANES III were recalibrated to standardized creatinine measurements obtained at the Cleveland Clinic Research Laboratory (Cleveland, OH, USA) as standard creatinine = $0.184+0.960 \times$ measured serum creatinine [22]. Random spot urine samples were obtained and frozen. Urine albumin was measured using a solid-phase fluorescence immunoassay, and urine creatinine was measured using the modified Jaffé kinetic method in the same laboratory. Estimated glomerular filtration rate (eGFR) was calculated through determination of SCr using the Chronic Kidney Disease Epidemiology Collaboration (CKD-EPI) equation [23]. Albuminuria, which is calculated as the urinary albumin-tocreatinine ratio (UACR), is expressed as milligrams of albumin per gram of creatinine $(\mathrm{mg} / \mathrm{g} \mathrm{Cr})$ using American Diabetes Association categories: normal ( $<30 \mathrm{mg} / \mathrm{g} \mathrm{Cr})$, and albuminuria $(\geq 30 \mathrm{mg} / \mathrm{g}$ Cr) [24]. We defined the stages of CKD at baseline according to the National Kidney Foundation Kidney Disease Outcomes Quality Initiative CKD classification [25] based on the level of kidney function (eGFR) and the presence or absence of kidney damage (albuminuria). CKD stages 1 and 2 was defined by single assessment of albuminuria (i.e., no estimation of persistence) and eGFR $\geq 90$ and $60-89 \mathrm{~mL} / \mathrm{min} / 1.73 \mathrm{~m}^{2}$, respectively, while stages 3 and 4 were defined as $30-59$ and $15-29 \mathrm{~mL} / \mathrm{min} / 1.73 \mathrm{~m}^{2}$, respectively.

\section{Outcomes}

The development of ESKD, that is, initiation of maintenance dialysis or kidney transplantation, was defined as entry into the USRDS) Registry from the time of enrollment into NHANES III through December 31, 2008. ESKD data are available for those NHANES III participants who agreed to provide personal identification data to NCHS and whom NCHS was able to match with
USRDS administrative records [26]. Mortality was ascertained through the National Death Index [27] starting from the time of enrollment and followed through December 31, 2008. Mortality status for NHANES III survey participants was ascertained primarily through probabilistic record matching with the NDI. All participants with sufficient identifying data were eligible for mortality linkage. Those participants whose records did not meet the minimum data requirements were ineligible for the mortality linkage, and this resulted in a loss of sample size. We censored all the data up to December 31, 2008, to keep the time frames equal.

\section{Statistical Analysis}

All continuous variables were tested for normality before statistical analysis. The Kolmogorov-Smirnov test was performed to determine if normality of the distribution of the parameters is consistent with the data. Comparisons between groups were performed using analysis of variance with a normal distribution and $\chi^{2}$ test or Fisher's exact test for categorical variables. Data that did not show a normal distribution were compared using KruskalWallis test. FVs intake was categorized into selected categories; $<2$, 2 to $<3,3$ to $<4,4$ to $<6$, and $\geq 6$ times/day. A $p$ value for the crude dose-response association of each sociodemographic or clinical variable (outcome) across selected categories of FV intake was obtained using a linear regression model on the categories' median values for numeric data (e.g., mean age) and ordinal logistic regression on the categories' median values for categorical data (e.g., proportions of current and past smokers).

Lower FV intake has been linked with a higher risk of mortality related to CVD. From data available from several epidemiological studies, it is obvious that patients with CKD are more prone to develop CVD and CVD kills people with CKD more frequently and prematurely [28]. We used the Fine Gray competing risk method with death from CVD as the competing event to explore the relation (relative hazard $[\mathrm{RH}]$ and $95 \%$ confidence interval [CI]) between FV intake, comparing each FV category with reference category ( $\geq 6$ times/day, the highest intake), and rate of ESKD events. Korn and Graubard [29] CI was calculated for the risk estimates. Confounders considered to be clinically related to the exposure and outcome were included in multivariable models. The model was adjusted for age, sex, race/ethnicity, SES, meat and fish intake, HbA1C, SBP, eGFR, and UACR, all measured at baseline. Prior literature $[18,19]$ has demonstrated SBP imparting highest risk of decline in kidney function compared to diastolic BP, pulse pressure, and mean arterial pressure; therefore, we adjusted our model for SBP. Tests for trend were performed using median intake values in the selected categories as continuous variables in competing risk analysis. Additional competing risk models were run to test the robustness of the findings in those with manifest CKD of any severity (stages $1-4$ of severity), and then separately in those with stages 3-4 of severity.

\section{Cause-Specific Hazards Model}

Multivariable cause-specific hazards models were used to assess the association of FV intake with development of ESKD in the general population and subsequently in adults with established CKD (i.e., any CKD and advanced/severe CKD). The models were adjusted for age, sex, race/ethnicity, SES, meat and fish intake, HbA1C, SBP, eGFR, and UACR, all measured at baseline. 
Table 2. Baseline characteristics according to the selected categories of fruit and vegetable intake in US adults in NHANES III $(n=14,725)$

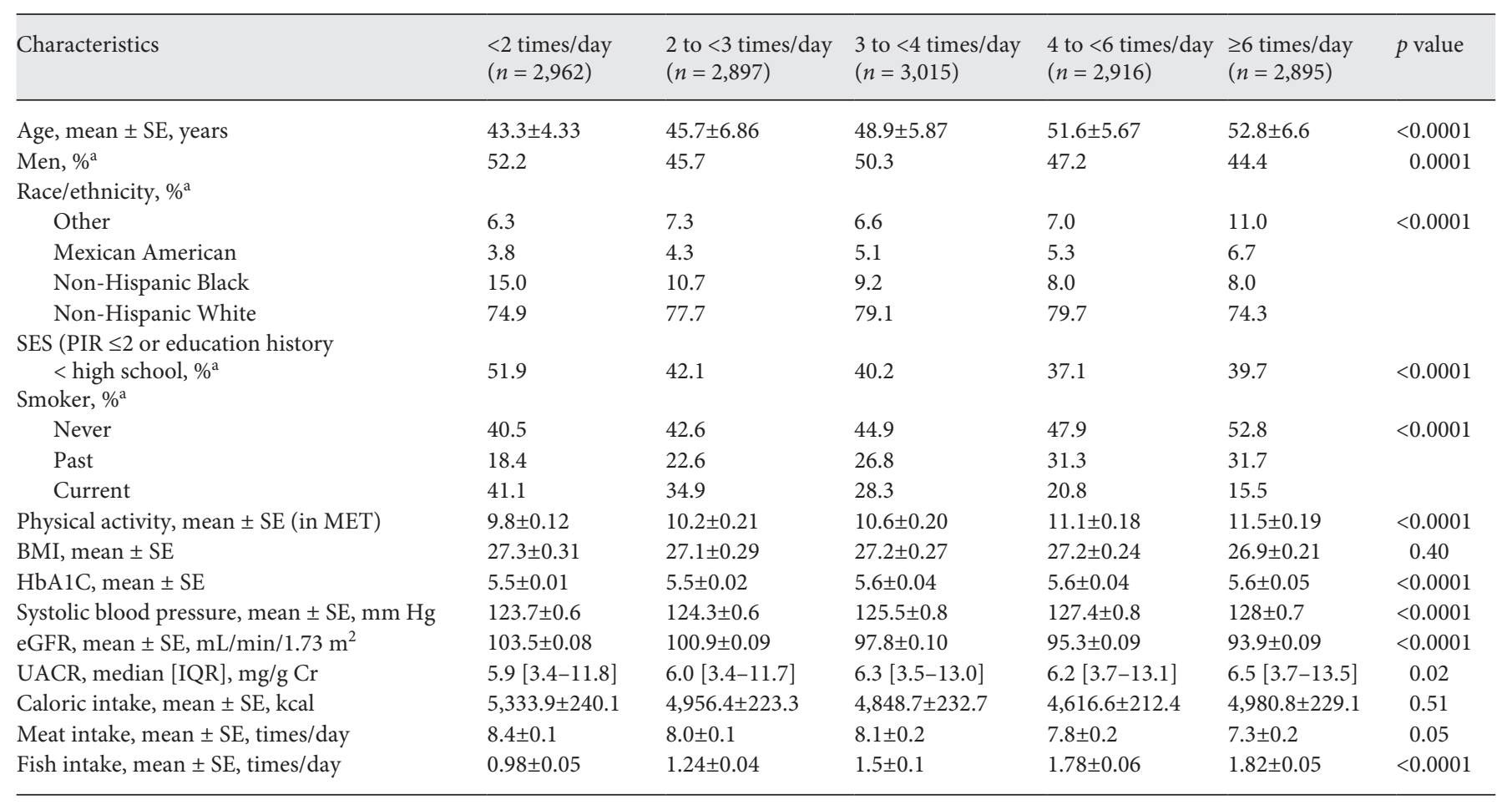

This information was based on the results from linking NHANES III, participant data to USRDS, administrative records and to the National Death Index (NDI) death certificate records. SES, socioeconomic status; PIR, poverty income ratio; UACR, urine albumin-creatinine ratio; eGFR, estimated glomerular filtration rate; BMI, body mass index; SE, standard error; IQR, interquartile range; NHANES III, Third National Health and Nutrition Examination Survey;

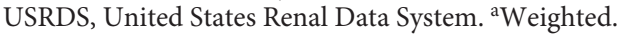

Adjusted dietary weights and balanced repeated replication weights accounting for linkage eligibility were produced by fitting a marginal model. These weights were then used in the survey procedures as the final weights following the analytical guidelines for NHANES III data [30, 31]. Results were considered significant if $p<0.05$. All analyses were performed using SAS 9.4 (SAS Institute, Inc., Cary, NC, USA).

\section{Results}

\section{Baseline Characteristics}

Baseline characteristics of participants $(n=14,725)$ according to selected categories of FV intake are reported in Table 2. FV intake was categorized into selected categories; $<2,2$ to $<3,3$ to $<4,4$ to $<6$, and $\geq 6$ times/day. Mean age at baseline was $50.1 \pm 0.8$ years, and $49.3 \%$ were men. Mean eGFR was $100.1 \pm 14.2 \mathrm{~mL} / \mathrm{min} / 1.73 \mathrm{~m}^{2}$. The median FV intake was 3.6 times/day. Participants consuming FVs $\geq 6$ times/day (highest FV intake) were older and more likely to be women, had a lesser proportion of participants with lower poverty income ratio and lower levels of education than those in the lowest quintile. Additionally, those eating FVs $\geq 6$ times/day had higher HbA1C, higher systolic blood pressure, and lower BMI than those eating $<2$ times/day.

\section{Association of FV Intake with Risk of ESKD in All Adult NHANES III Participants}

Over a median follow-up period of up to 9.2 years, $1.5 \%$ of participants $(n=230)$ developed ESKD and 9.3\% of participants $(n=1,370)$ died due to CVD. In crude analysis (model 1), the risk of ESKD was higher across the categories of FV intake when compared with $\geq 6$ times/day (Table 3a). In the fully adjusted model (model 6), there remained a graded relationship ( $p$ for linear trend $=0.01)$. Compared to the highest FV intake, participants with $<2$ times/day $(\mathrm{RH}[95 \% \mathrm{CI}]=$ (1.45 [1.24-1.68]), those with 2 to $<3$ times/day $(1.40$ $[1.18-1.61])$, and those with 3 to $<4$ times/day $(1.25$ [1.04-1.46)] remained at significantly higher ESKD risk (Fig. 1a; Table 3a). A test for interaction in the fully adjusted model showed that the inverse association be- 
Table 3. Association of FV intake with ESKD using age as a time scale and cardiovascular mortality as a competing risk in (a) the general population $(n=14,725$, ESKD events $=230$, CVD death $=1,370)$, (b) those with CKD stages $1-4(n=5,346, \mathrm{ESKD}$ events $=180, \mathrm{CVD}$ death $=1,037)$, and $(c)$ those with CKD stages $3-4(n=1,084$, ESKD events $=120$, CVD death $=239)$

\begin{tabular}{|c|c|c|c|c|c|}
\hline & \multicolumn{5}{|l|}{ RH (95\% CI) } \\
\hline \multicolumn{6}{|c|}{ a General population } \\
\hline Model 1 & $1.82(1.57-2.06)$ & $1.68(1.38-1.94)$ & $1.61(1.29-1.90)$ & $1.31(1.03-1.55)$ & 1.0 (ref.) \\
\hline Model 2 & $1.78(1.52-1.99)$ & $1.60(1.41-1.87)$ & $1.60(1.24-1.87)$ & $1.33(1.10-1.59)$ & 1.0 (ref.) \\
\hline Model 3 & $1.72(1.54-1.92)$ & $1.69(1.46-1.96)$ & $1.55(1.20-1.84)$ & $1.42(1.20-1.65)$ & 1.0 (ref.) \\
\hline Model 6 & $1.45(1.24-1.68)$ & $1.40(1.18-1.61)$ & $1.25(1.04-1.46)$ & $1.14(0.97-1.35)$ & 1.0 (ref.) \\
\hline \multicolumn{6}{|c|}{ b CKD stages 1-4 } \\
\hline Model 1 & $1.78(1.53-2.00)$ & $1.70(1.51-1.93)$ & $1.55(1.38-1.80)$ & $1.50(1.25-1.72)$ & 1.0 (ref.) \\
\hline Model 2 & $1.72(1.56-1.96)$ & $1.72(1.55-1.91)$ & $1.52(1.30-1.71)$ & $1.46(1.22-1.70)$ & 1.0 (ref.) \\
\hline Model 3 & $1.69(1.50-1.88)$ & $1.68(1.43-1.85)$ & $1.44(1.25-1.70)$ & $1.43(1.21-1.67)$ & 1.0 (ref.) \\
\hline \multicolumn{6}{|c|}{ c CKD stages 3-4 } \\
\hline Model 1 & $1.35(1.13-1.54)$ & $1.59(1.38-1.79)$ & $1.49(1.28-1.75)$ & $1.32(1.08-1.54)$ & 1.0 (ref.) \\
\hline Model 2 & $1.33(1.10-1.52)$ & $1.55(1.37-1.74)$ & $1.46(1.24-1.71)$ & $1.30(1.11-1.51)$ & 1.0 (ref.) \\
\hline Model 3 & $1.22(1.02-1.43)$ & $1.53(1.25-1.70)$ & $1.40(1.20-1.64)$ & $1.31(1.10-1.51)$ & 1.0 (ref.) \\
\hline Model 4 & $1.17(1.04-1.38)$ & $1.49(1.29-1.71)$ & $1.36(1.16-1.58)$ & $1.27(1.07-1.46)$ & 1.0 (ref.) \\
\hline Model 5 & $1.15(1.01-1.35)$ & $1.44(1.25-1.68)$ & $1.29(1.10-1.48)$ & $1.16(1.00-1.35)$ & 1.0 (ref.) \\
\hline Model 6 & $1.12(1.00-1.33)$ & $1.39(1.18-1.60)$ & $1.21(1.05-1.42)$ & $1.04(0.93-1.25)$ & 1.0 (ref.) \\
\hline
\end{tabular}

Model 1, crude; model 2, model 1 + adjusted for age, gender, race/ethnicity; model 3, model 2 + adjusted for socio-economic status; model 4, model 3 + adjusted for meat and fish intake; model 5, model 4 + adjusted for HbA1C, systolic blood pressure; model 6, model 5 + adjusted for eGFR, UACR. RH, relative hazard; ESKD, end-stage kidney disease; CVD, cardiovascular disease; CKD, chronic kidney disease; eGFR, estimated glomerular filtration rate; UACR, urinary albumin-to-creatinine ratio; FV intake, fruit and vegetable intake.

tween FV intake and CKD status was stronger for persons with CKD than for persons without CKD ( $p$ for interaction $=0.03$ ).

\section{Risk of ESKD in NHANES III Adult Participants with CKD at Baseline}

A total of 5,346 NHANES III participants were classified as having CKD stages $1-4$ based on eGFR and albuminuria measures. Their general characteristics are shown in online suppl. Table 1; for all online suppl. material, see www.karger.com/doi/10.1159/000514754. A total of $8.8 \%$ of this sample $(n=180)$ progressed to ESKD and $19.4 \%(n=1,037)$ died due to CVD. In fully adjusted models, we observed a higher ESKD risk across decreasing categories of FV intake ( $p$ for trend $=0.05$ ). Compared to the highest intake, eating $\mathrm{FV}<2$ times/day (1.41 [1.18$1.62]), 2$ to $<3$ times/day (1.46 [1.25-1.69]), and 3 to $<4$ times/day (1.19 [1.00-1.36]) were all associated with a statistically significant higher ESKD risk (Fig. 1b; Table 3 b model 6).

\section{ESKD Incidence in Participants with CKD Stage 3-4 at Baseline}

A total of 1,084 NHANES III participants were classified as having CKD stages 3-4. Their general characteristics are shown in online suppl. Table 2. During followup, 120 (11.1\%) participants developed ESKD and 239 (22.1\%) died due to CVD. In this group, we noted a nonlinear trend across FV quintiles and ESKD incidence in each of the models (Table 3c). The RHs were substantially attenuated with the adjustment for the clinical factors (i.e., HbA1C, systolic blood pressure, model 5) across the categories of FV intake. In the fully adjusted model (model 6), compared to highest intake, those in lowest categories of FV intake had a greater $\mathrm{RH}$ of ESKD - for 4 to $<6$ times/day: 1.04 [0.93-1.25], for 3 to 
Fig. 1. Association between intake of fruits and vegetables per day and risk of ESKD in all adults $(n=14,725$, ESKD events $=230$, cardiovascular death $=1,370)(\mathbf{a})$, all stages of CKD ( $n=5,346$, ESKD events $=180$, cardiovascular death $=1,037)(\mathbf{b})$, and stage 3 or 4 CKD $(n=1,084$, ESKD events $=120$, cardiovascular death $=239)(\mathbf{c})$ using age as a time scale and cardiovascular mortality as a competing risk. The model is adjusted for age, gender, race/ethnicity, socioeconomic status, meat and fish intake, HbA1C, systolic blood pressure, eGFR, and UACR. ESKD, end-stage kidney disease; CKD, chronic kidney disease; eGFR, estimated glomerular filtration rate; UACR, urinary albumin-to-creatinine ratio; $\mathrm{RH}$, relative hazard; CI, confidence interval.

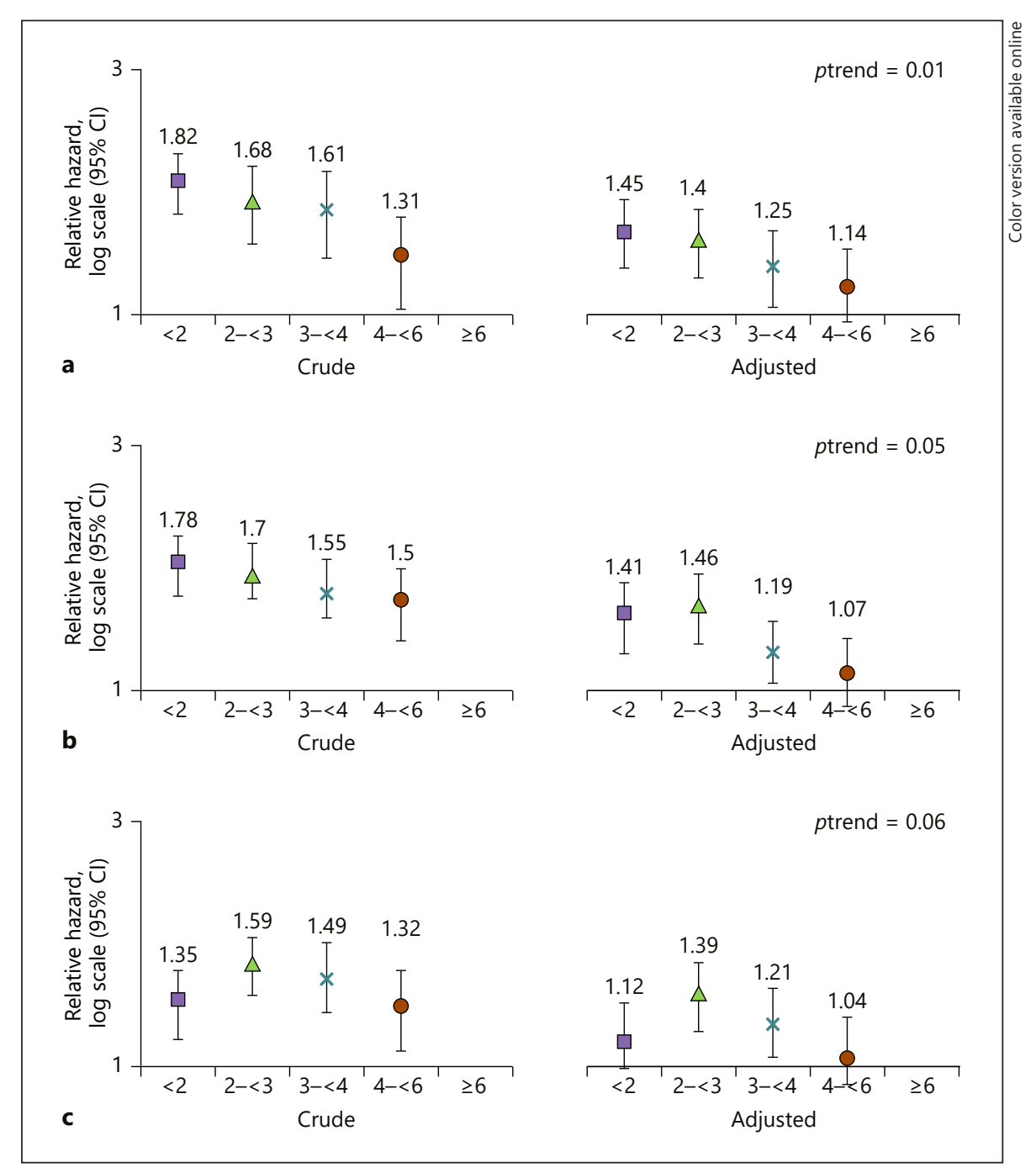

$<4$ times/day: 1.21 [1.05-1.42], for 2 to $<3$ times/day: 1.39 [1.18-1.60], and for <2 times/day: 1.12 [1.00-1.33] (Fig. 1c; Table 3c).

\section{Cause-Specific Hazards Model}

The multivariable cause-specific hazards model yielded similar results compared with those estimated using the competing risk model in all the 3 groups (data not shown). In the general population, the RH of ESKD in the fully adjusted model was $1.38(1.12-1.53)$ for $<2$ times/ day, $1.31(1.10-1.49)$ for 2 to $<3$ times/day, 1.23 (1.03$1.40)$ for 3 to $<4$ times/day, and $1.17(0.97-1.33)$ for 4 to $<6$ times/day. The RH of ESKD in the fully adjusted model in adults with CKD was $1.36(1.16-1.58)$ for $<2$ times/ day, $1.40(1.19-1.61)$ for 2 to $<3$ times/day, 1.21 (1.00$1.37)$ for 3 to $<4$ times/day, and $1.09(0.93-1.24)$ for 4 to $<6$ times/day. Last, in adults with CKD stage 3-4, the RH of ESKD was $1.19(1.01-1.40)$ for $<2$ times/day, 1.41 (1.16-1.63) for 2 to $<3$ times/day, 1.25 (1.03-1.48) for 3 to $<4$ times/day, and $1.07(0.95-1.34)$ for 4 to $<6$ times/ day.

\section{Discussion}

This study demonstrates a consistent inverse association between daily very low intake ( $<3$ times) of FVs and risk of ESKD among adult NHANES III participants, suggesting daily higher intake of FVs may be effective in preventing progression to ESKD. Our observation of an association between higher FV intake and low ESKD risk in the general US population align with a Korean study [32] reporting higher risk of incident CKD (development of eGFR $<60 \mathrm{~mL} / \mathrm{min} / 1.73 \mathrm{~m}^{2}$ during follow-up) and lower 
acid load among participants consuming low amounts of FVs. Collectively, they both expand previous studies evaluating the adherence of dietary patterns rich in plantbased foods and CKD risk [9]. For instance, in the Atherosclerosis Risk in Communities cohort, a greater adherence to various plant-based diet indices was consistently associated with the risk of incident CKD [33]. In a prospective observational Northern Manhattan Study $(N=$ 900 ), plant-based diets were associated with a $12 \%$ lower risk of decline in eGFR compared with meat-based diets [34]. Mediterranean diet patterns and Dietary Approaches to Stop Hypertension diet patterns, which also emphasize the intake of FVs, have been associated with a lower risk of incident CKD and ESKD [35, 36].

Our observation of a similar association between higher FVs and low ESKD risk in individuals with manifest CKD is interesting; we are not aware of any study that has addressed this before. Four well-designed RCTs by Goraya et al. $[12,13,37,38]$ have demonstrated that FVs provide a comparable benefit to sodium bicarbonate by improving metabolic acidosis and GFR preservation in people with nondialysis CKD. The largest of these studies [13] involved 108 patients with macroalbuminuria and CKD stage 3 randomized for 3 years to usual care or interventions designed to reduce dietary acid by $50 \%$, using either sodium bicarbonate or alkali-rich FVs. Both interventions reduced urine excretion of angiotensinogen and preserved eGFR similarly and to a greater extent than usual care [38]. Owing to hyperkalemia fears, this trial excluded individuals with diabetes with plasma $\mathrm{K}$ levels $>4.6 \mathrm{mEq} / \mathrm{L}$. Our results thus agree with those of this trial and expand the finding to unselected persons with CKD.

There are various mechanisms by which a diet rich in FVs may reduce ESKD risk. One of them may involve the promotion of body's alkalinity. On one hand, the high consumption of meat and refined cereals in modern society is associated with lower urine $\mathrm{pH}$ and higher serum bicarbonate [39] more so in the presence of comorbid CKD $[40,41]$. However, the intake of FVs, which are net alkalinizing in nature, may neutralize acidosis and its consequences [42]. The predominant anions in FVs are citrate and malate, and when metabolized, they release bicarbonate and thus contribute alkali to the body. In healthy kidneys, excess dietary acid is neutralized and eliminated in the kidneys. But in CKD, the ability to excrete both acid and ammonia, a by-product of the neutralization of acid, is significantly impaired. As such, metabolic acidosis is frequent in persons with CKD and is a well-established risk factor for a faster disease progression [43].
Other mechanisms may involve the variety of healthy nutrients and phytochemicals - including fiber, vitamin C, carotenoids, antioxidants, potassium, and flavonoids which are present in FVs and may act separately or synergistically in reducing inflammation and oxidative stress [44]. Intake of dietary fiber and FVs has been shown to reduce cholesterol levels, blood pressure, inflammation, and platelet aggregation, and to improve vascular and immune function [45-48]. Furthermore, fiber intake promotes the growth of saccharolytic bacteria [45], which produce short chain fatty acids (alkali) and other antiinflammatory compounds, and lower the generation of uremic toxins [44]. Uremic toxins, such as P-cresol or indoxyl sulfate, are involved in the onset and progression of CKD through promotion of fibrogenesis in the kidney [49]. Antioxidants in FVs may neutralize reactive oxygen species and reduce DNA damage [46], glucosinolates in cruciferous vegetables induce detoxifying enzymes [47] and intake of FVs may also modulate steroid hormone concentrations and hormone metabolism [47]. A high FV intake may also reduce risk of progression to ESKD indirectly, by displacement of unhealthy foods high in saturated fat, trans fat, glycemic load, and sodium.

We observe that though the risk of ESKD in participants with CKD stages 3-4 in the lowest category of intake was attenuated, the statistical significance almost disappeared (1.12 [1.00-1.33]). We attribute this loss of statistical significance to potentially other unmeasured aspects of health status in these participants with advanced CKD (E-value [50] for the risk estimate $=1.49$ when taking into account unmeasured confounders), smaller sample size, and the possibility of linkage eligibility bias; however, this should have been mitigated with the weight adjustments. We note in this regard that they were older, had higher systolic blood pressure, and did not engage in intense physical activity compared to other adults with higher eGFR in our study. Additionally, it is possible that participants at this stage of CKD severity may have received dietary advice to reduce their FV intake to prevent hyperkalemia.

Strengths of this study include a large sample size, nationally representative study population, and long-term follow up. The evaluation of ESKD, the consequential end point in CKD progression, is another strength. Several limitations of our study should be considered. First, participants used an FFQ to report their dietary intakes, which can be subject to recall bias and social desirability bias. However, trained interviewers administered FFQs using visual aids to minimize error. Second, results are representative of the US population in the early 90s, and extrapola- 
tion to other periods or countries should be done with caution as diet is determined by many sociocultural factors. Because diet quality has deteriorated over time toward lower intake of fruits and vegetables and higher intake of meat and processed foods $\left(\beta=-1.53\right.$; $p_{\text {trend }}<0.001$ for intake of fruits and vegetables, calculated using the food frequency questionnaire, over the years 1994-2006), we speculate the magnitude of observed risk to be potentially higher in the current times. Our findings of a decline in the intake of fruits and vegetables over the period is consistent with the results shown by USDA Economic Research Service researchers who linked the loss-adjusted food availability data and data from 6 national food intake surveys conducted from 1994-1998 to 2007-2008 [51]. Additionally, the laboratory data were also collected long ago. Future prospective studies with periodic/longitudinal data on potassium content and form of the diet (cooked, canned, frozen or fresh fruit and vegetables), metabolic parameters, disease status, as well as other healthy lifestyles are warranted. Third, results are based on one dietary assessment per participant, and may not accurately represent longterm dietary habits. Additionally, the laboratory data were also collected at a single time point. Our confounders were assessed at baseline, and the development of diabetes or hypertension during follow-up (which are known risk factors for ESKD) may also explain our findings. Fourth, there are very few ESKD events and the possibility of linkage eligibility bias in NHANES III. Last, as it is the case for all observational analyses, causality cannot be inferred.

To conclude, low intake of FVs was associated with higher risk of ESKD in US adults with and without CKD, supporting an emerging body of literature on the potential benefits of plant-rich diets for primary and secondary CKD prevention. In the absence of trial evidence, our findings can have clinical implications and may inform patient-centered discussions in clinical practice. In persons with manifest $\mathrm{CKD}$, our results complement recent trial evidence [33] and challenge the well-meaning guidance to limit FV intake to prevent higher dietary potassium load, which may deprive CKD patients, even those with stage 3 or $4 \mathrm{CKD}$, of the potential benefits of these foods. Further research, particularly on the risks of diet-induced hyperkalemia in advanced CKD patients, is warranted.

\section{Acknowledgements}

We thank the participants and staff of the National Health and Nutrition Examination Survey.

The Establishing a Surveillance System for Chronic Kidney Disease project is supported under a cooperative agreement from the Centers for Disease Control and Prevention through Grant No. NU58DP003836-05-01. Dr. Carrero acknowledges support from the Swedish Research Council (\#2019-01059),

The findings and conclusions in this report are those of the authors and do not necessarily represent the official position of the Centers for Disease Control and Prevention.

The CDC CKD Surveillance Team consists of group members led by University of California, San Francisco (Neil Powe [Principal Investigator], Tanushree Banerjee, Delphine Tuot, Chi-yuan Hsu, Charles McCulloch, Deidra Crews, Raymond Hsu, Vanessa Grubbs, Kirsten Bibbins-Domingo, Michael Shlipak, Carmen Peralta, Anna Rubinsky, Josef Coresh, Joanne Rodrigue); University of Michigan (Rajiv Saran [Principal Investigator], Vahakn Shahinian, Brenda Gillespie, Hal Morgenstern, Michael Heung, William Herman, Jennifer Bragg-Gresham, Diane Steffick, Anca Tilea, Maggie Yin, Ian Robinson, Kara Zivin, Vivian Kurtz, April Wyncott); and the CDC (Nilka Ríos Burrows [Technical Advisor], Mark Eberhardt, Juanita Mondesire, Priti Patel, Meda Pavkov, Deborah Rolka, Sharon Saydah,, Larry Waller).

\section{Statement of Ethics}

This study is approved by the University of California, San Francisco Institutional Review Board (Committee on Human Research application \#10-04162) and by the Research Data Center of the National Center of Health Statistics (\#1508).

\section{Conflict of Interest Statement}

No financial disclosures were reported by the authors of this paper.

\section{Author Contributions}

T.B., J.J.C., and N.R.P. were responsible for concept, analytic design, data analysis, interpretation of results, and manuscript preparation. C.E.M. helped with the interpretation of results and preparation of manuscript. H.M., R.S., N.R.B., and K.S. helped with the interpretation of results, gave thoughtful comments, and critically reviewed the manuscript.

\section{References}

1 Xu H, Sjögren P, Ärnlöv J, Banerjee T, Cederholm T, Risérus U, et al. A proinflammatory diet is associated with systemic inflammation and reduced kidney function in elderly adults. J Nutr. 2015;145(4):729-35.

2 Jha V, Garcia-Garcia G, Iseki K, Li Z, Naicker S, Plattner B, et al. Chronic kidney disease: global dimension and perspectives. Lancet. 2013;382(9888):260-72.

3 Levin A, Tonelli M, Bonventre J, Coresh J, Donner JA, Fogo AB, et al. Global kidney health 2017 and beyond: a roadmap for closing gaps in care, research, and policy. Lancet. 2017;390(10105):1888-917. 
4 Tohidi M, Hasheminia M, Mohebi R, Khalili D, Hosseinpanah F, Yazdani B, et al. Incidence of chronic kidney disease and its risk factors, results of over 10 year follow up in an Iranian cohort. PLoS One. 2012;7(9):e45304.

5 Kazancioglu R. Risk factors for chronic kidney disease: an update. Kidney Int Suppl (2011). 2013;3(4):368-71.

6 Chobanian AV, Bakris GL, Black HR, Cushman WC, Green LA, Izzo JL, et al. The seventh report of the joint national committee on prevention, detection, evaluation, and treatment of high blood pressure: the JNC 7 report. JAMA. 2003;289(19):2560-72.

7 Chen ST, Maruthur NM, Appel LJ. The effect of dietary patterns on estimated coronary heart disease risk: results from the Dietary Approaches to Stop Hypertension (DASH) trial. Circ Cardiovasc Qual Outcomes. 2010; 3(5):484-9.

8 Chauveau P, Aparicio M, Bellizzi V, Campbell $\mathrm{K}$, Hong X, Johansson L, et al. Mediterranean diet as the diet of choice for patients with chronic kidney disease. Nephrol Dial Transplant. 2018;33(5):725-35.

9 Bach KE, Kelly JT, Palmer SC, Khalesi S, Strippoli GFM, Campbell KL. Healthy dietary patterns and incidence of CKD: a meta-analysis of cohort studies. Clin J Am Soc Nephrol. 2019 Oct 7;14(10):1441-9.

10 Kelly JT, Palmer SC, Wai SN, Ruospo M, Carrero JJ, Campbell KL, et al. Healthy dietary patterns and risk of mortality and ESRD in CKD: a meta-analysis of cohort studies. Clin J Am Soc Nephrol. 2017;12(2):272-9.

11 Cases A, Cigarrán-Guldrís S, Mas S, Gonzalez-Parra E. Vegetable-based diets for chronic kidney disease? It is time to reconsider. Nutrients. 2019 Jun 4;11(6): 1263.

12 Goraya N, Simoni J, Jo CH, Wesson DE. A comparison of treating metabolic acidosis in CKD stage 4 hypertensive kidney disease with fruits and vegetables or sodium bicarbonate. Clin J Am Soc Nephrol. 2013;8(3):371-81.

13 Goraya N, Simoni J, Jo CH, Wesson DE. Treatment of metabolic acidosis in patients with stage 3 chronic kidney disease with fruits and vegetables or oral bicarbonate reduces urine angiotensinogen and preserves glomerular filtration rate. Kidney Int. 2014;86(5): 1031-8.

14 Golden C, Driscoll AK, Simon AE, Judson DH, Miller EA, Parker JD, et al. Linkage of NCHS population health surveys to administrative records from Social Security Administration and Centers for Medicare and Medicaid Services. Vital Health Stat 1. 2015 Sep; (58):1-53.

15 National Center for Health Statistics - Office of Analysis and Epidemiology. The Linkage of National Center for Health Statistics Survey Data to the National Death Index-2011 Linked Mortality File (LMF): Methodology Overview and Analytic Considerations. Hyattsville, Maryland; 2019 Mar.

16 Anonymous. Plan and operation of the Third national health and nutrition examination survey, 1988-94. Series 1: programs and collection procedures. Vital Health Stat 1. 1994; (32):1-407

17 International Expert Committee. International Expert Committee report on the role of the $\mathrm{AlC}$ assay in the diagnosis of diabetes. Diabetes Care. 2009;32(7):1327-34.

18 Hunter Young J, Klag MJ, Paul M, Whyte JL, Pahor M, Coresh J. Blood pressure and decline in kidney function: findings from the Systolic Hypertension in the Elderly Program (SHEP). J Am Soc Nephrol. 2002 Nov; 13(11):2776-82. 19 Peralta CA, Hicks LS, Chertow GM, Ayanian JZ, Vittinghoff E, Lin F, et al. Control of hypertension in adults with chronic kidney disease in the United States. Hypertension. 2005; 45(6):1119-24.

20 US Department of Health and Human Services. Centers for Disease Control and Prevention MMWR. 2017 Nov 17;66(45):1247.

21 Dresser CM. From nutrient data to a data base for a health and nutrition examination survey. Organization, coding, and values-real or imputed. Proceedings of the Eighth National Data Base Conference. Minneapolis, MN, 1983 Jul.

22 Coresh J, Selvin E, Stevens LA, Manzi J, Kusek JW, Eggers P, et al. Prevalence of chronic kidney disease in the United States. JAMA. 2007; 298(17):2038-47.

23 Levey AS, Stevens LA, Schmid CH, Zhang YL, Castro AF 3rd, Feldman HI, et al. A new equation to estimate glomerular filtration rate. Ann Intern Med. 2009;150(9):604-12.

24 Molitch ME, DeFronzo RA, Franz MJ, Keane WF, Mogensen CE, Parving HH, et al. American Diabetes Association: nephropathy in diabetes. Diabetes Care. 2004;27(Suppl 1): S79-83.

25 National Kidney Foundation. K/DOQI clinical practice guidelines for chronic kidney disease: evaluation, classification, and stratification. Am J Kidney Dis. 2002;39(2 Suppl 1): S1-266.

26 Golden C, Driscoll AK, Simon AE, Judson DH, Miller EA, Parker JD, et al. Linkage of NCHS population health surveys to administrative records from Social Security Administration and Centers for Medicare \& Medicaid Services. Vital Health Stat 1. 2015 Sep;(58): $1-53$.

27 National Center for Health Statistics. The Third National Health and Nutrition Examination Survey (NHANES III) linked mortality file: matching methodology. Hyattsville, MD: National Center for Health Statistics; 2005.

28 Herzog CA, Asinger RW, Berger AK, Charytan DM, Díez J, Hart RG, et al. Cardiovascular disease in chronic kidney disease. A clinical update from Kidney Disease: Improving Global Outcomes (KDIGO). Kidney Int. 2011;80(6):572-86

29 Korn EL, Graubard BI. Wiley series in probability and statistics. Analysis of health surveys. New York: Wiley; 1999.

30 National Center for Health Statistics. Analytical and reporting guidelines: the third na- tional health and nutrition examination survey, 1988-1994. Hyattsville, MD: National Center for Health Statistics; 1996.

31 Fay RE. Theory and application of replicate weighting for variance calculations. In: Proceedings of the survey research methods section. American Statistical Association; 1989. p. 212-7.

32 Jhee JH, Kee YK, Park JT, Chang TI, Kang $\mathrm{EW}$, Yoo TH, et al. A diet rich in vegetables and fruit and incident CKD: a communitybased prospective cohort study. Am J Kidney Dis. 2019 Oct;74(4):491-500.

$33 \mathrm{Kim} \mathrm{H}$, Caulfield LE, Garcia-Larsen V, Steffen LM, Grams ME, Coresh J, et al. Plant-based diets and incident CKD and kidney function. Clin J Am Soc Nephrol. 2019;14(5):682-91.

34 Khatri M, Moon YP, Scarmeas N, Gu Y, Gardener $\mathrm{H}$, Cheung K, et al. The association between a Mediterranean-style diet and kidney function in the Northern Manhattan Study cohort. Clin J Am Soc Nephrol. 2014 Nov 7; 9(11):1868-75.

35 Rebholz CM, Crews DC, Grams ME, Steffen LM, Levey AS, Miller ER, et al. DASH (Dietary Approaches to Stop Hypertension) diet and risk of subsequent kidney disease. Am J Kidney Dis. 2016;68(6):853-61.

36 Gallieni M, Cupisti A. DASH and Mediterranean diets as nutritional interventions for CKD patients. Am J Kidney Dis. 2016 Dec; 68(6):828-30.

37 Goraya N, Simoni J, Jo C, Wesson DE. Dietary acid reduction with fruits and vegetables or bicarbonate attenuates kidney injury in patients with a moderately reduced glomerular filtration rate due to hypertensive nephropathy. Kidney Int. 2012;81(1):86-93.

38 Goraya N, Munoz-Maldonado Y, Simoni J, Wesson DE. Fruit and vegetable treatment of chronic kidney disease-related metabolic acidosis reduces cardiovascular risk better than sodium bicarbonate. Am J Nephrol. 2019; 49(6):438-48.

39 Welch AA, Mulligan A, Bingham SA, Khaw $\mathrm{KT}$. Urine $\mathrm{pH}$ is an indicator of dietary acidbase load, fruit and vegetables and meat intake: results from the European Prospective Investigation into Cancer and Nutrition (EPIC)- Norfolk Population Study. Br J Nutr. 2008;99:1335-43.

40 Scialla JJ, Appel LJ, Astor BC, Miller ER, Beddhu S, Woodward M, et al. Estimated net endogenous acid production and serum bicarbonate in African Americans with chronic kidney disease. Clin J Am Soc Nephrol. 2011; 6(7):1526-32.

41 Banerjee T, Crews DC, Wesson DE, Tilea A, Saran R, Rios Burrows N, et al. Dietary acid load and chronic kidney disease among adults in the United States. BMC Nephrol. 2014;15, 137.

42 Ausman LM, Oliver LM, Goldin BR, Woods MN, Gorbach SL, Dwyer JT. Estimated net acid excretion inversely correlates with urine $\mathrm{pH}$ in vegans, lacto-ovo vegetarians, and omnivores. J Ren Nutr. 2008;18(5):456-65. 
43 Chen W, Abramowitz MK. Metabolic acidosis and the progression of chronic kidney disease. BMC Nephrol. 2014;15:55.

44 Carrero JJ, González-Ortiz A, Avesani CM, Bakker SJL, Bellizzi V, Chauveau P, et al. Plant-based diets to manage the risks and complications of chronic kidney disease. Nature Rev Nephrol. 2020 Sep;16(9):525-42.

45 Anderson JW, Baird P, Davis RH Jr, Ferreri S, Knudtson M, Koraym A, et al. Health benefits of dietary fiber. Nutr Rev. 2009;67(4):188205.

46 Lampe JW. Health effects of vegetables and fruit: assessing mechanisms of action in human experimental studies. Am J Clin Nutr. 1999;70(3 Suppl 1):475S-490S.

47 Broekmans WM, Klöpping-Ketelaars IA, Schuurman CR, Verhagen H, van den Berg H, Kok FJ, et al. Fruits and vegetables increase plasma carotenoids and vitamins and decrease homocysteine in humans. J Nutr. 2000; 130(6):1578-83.

48 Macready AL, George TW, Chong MF, Alimbetov DS, Gin Y, Vidal A, et al. Flavonoid-rich fruit and vegetables improve microvascular reactivity and inflammatory status in men at risk of cardiovascular disease: FLAVURS: a randomized controlled trial. Am J Clin Nutr. 2014;99:479-89.

49 Niwa T. Role of indoxyl sulfate in the progression of chronic kidney disease and cardiovascular disease: experimental and clinical effects of oral sorbent AST-120. Ther Apher Dial. 2011;15(2):120-4.
50 VanderWeele TJ, Peng D. Sensitivity analysis in observational research: introducing the $\mathrm{E}$ value. Ann Intern Med. 2017 Aug 15;167(4): $268-74$.

51 Lin B-H, Buzby JC, Anekwe TD, Bentley J. U.S. food commodity consumption broken down by demographics, 1994-2008. USDA, Economic Research Service; 2016.

52 Saglimbene VM, Wong G, Ruospo M, Palmer SC, Garcia-Larsen V, Natale P, et al. Fruit and vegetable intake and mortality in adults undergoing maintenance hemodialysis. Clin J Am Soc Nephrol. 2019;14(2):250-60.

53 Wang X, Ouyang Y, Liu J, Zhu M, Zhao G, Bao W, et al. Fruit and vegetable consumption and mortality from all causes, cardiovascular disease, and cancer: systematic review and dose-response meta-analysis of prospective cohort studies [published correction appears in BMJ. 2014;349:5472]. BMJ. 2014;349: g4490.

54 Tucker KL, Hallfrisch J, Qiao N, Muller D, Andres R, Fleg JL. The combination of high fruit and vegetable and low saturated fat intakes is more protective against mortality in aging men than is either alone: the Baltimore Longitudinal Study of Aging. J Nutr. 2005; 135(3):556-61.
55 Steffen LM, Jacobs DR, Stevens J, Shahar E, Carithers T, Folsom AR. Associations of whole-grain, refined-grain, and fruit and vegetable consumption with risks of all-cause mortality and incident coronary artery disease and ischemic stroke: the Atherosclerosis Risk in Communities (ARIC) Study. Am J Clin Nutr. 2003;78(3):383-90.

56 Bazzano LA, He J, Ogden LG, Loria CM, Vupputuri S, Myers L, et al. Fruit and vegetable intake and risk of cardiovascular disease in US adults: the first National Health and Nutrition Examination Survey Epidemiologic Followup Study. Am J Clin Nutr. 2002;76(1):93-9.

57 Bellavia A, Larsson SC, Bottai M, Wolk A, Orsini N. Fruit and vegetable consumption and all-cause mortality: a dose-response analysis. Am J Clin Nutr. 2013;98(2):454-9.

58 Leenders M, Sluijs I, Ros MM, Boshuizen HC, Siersema PD, Ferrari P, et al. Fruit and vegetable consumption and mortality: European prospective investigation into cancer and nutrition. Am J Epidemiol. 2013;178(4):590602 .

59 Colditz GA, Branch LG, Lipnick RJ, Willett WC, Rosner B, Posner BM, et al. Increased green and yellow vegetable intake and lowered cancer deaths in an elderly population. Am J Clin Nutr. 1985;41(1):32-6. 